
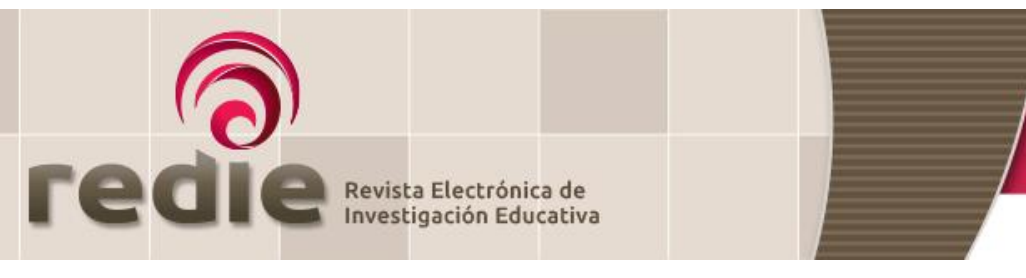

Vol. 21, 2019/e12

\title{
Aprendizaje basado en juegos formativos: caso Universidad en Colombia
}

\section{Learning Based on Educational Games: Case University in Colombia}

\author{
Oscar Alonso Vélez (1) osvelez@udem.edu.co \\ Sandra Milena Palacio López (1) spalacio@udem.edu.co \\ Yuri Lorene Hernández Fernández (1) yhernandez@udem.edu.co \\ Paola Andrea Ortiz Rendón (2) portiz@esumer.edu.co \\ Luisa Fernanda Gaviria Martínez (1) lugaviria@udem.edu.co \\ (1) Universidad de Medellín \\ (2) Institución Universitaria ESUMER \\ (Recibido: 5 de junio de 2017; Aceptado para su publicación: 16 de agosto de 2017)
}

Cómo citar: Vélez, O., Palacio, S. M., Hernández, Y. L., Ortiz, P. A. y Gaviria, L. F. (2019). Aprendizaje basado en juegos formativos: caso Universidad en Colombia. Revista Electrónica de Investigación Educativa, 21, e12, 1-10. doi:10.24320/redie.2019.21.e12.2024

\section{Resumen}

Las nuevas generaciones requieren un modelo de aprendizaje más dinámico, por lo que las Instituciones de Educación Superior han comenzado a articular sus herramientas pedagógicas a la enseñanza de las diferentes áreas del saber. El objetivo de este artículo es diseñar un juego gerencial para apoyar las asignaturas que contemplen dentro de su micro-currículo la temática de auditoria de marketing, utilizando una metodología exploratoria y descriptiva, apoyada en el aprendizaje basado en juegos. Para ello se realizó una revisión de la literatura y se llevó a cabo una prueba piloto con estudiantes de pregrado de una universidad colombiana. Los resultados muestran que el diseño del juego debe utilizar una estrategia interactiva, que a su vez guarde cierto tipo de complejidad y competitividad, adicionalmente se reveló que el juego refuerza elementos presentes en la temática estudiada.

Palabras clave: Estrategias de aprendizaje, juego educativo, marketing, auditoría de gestión.

\section{Abstract}

New generations require a more dynamic learning process, that's why high education institutions have begun to articulate new pedagogical tools in the different areas of knowledge. The objective of this article is to design and educational game to support the learning process of the areas related to marketing inside the micro-curriculum of a Colombian University. To accomplish this aim, a literature review was carried out and a pilot test was applied to pre-grade students. The results show that this type of games require of a mode interactive design in terms of complexity and competitiveness. Finally shows that this type of games improved knowledge in the simple tested. 


\section{Introducción}

Cada vez más el entorno demanda a las Instituciones de Educación Superior (IES) profesionales una formación que ayude a adaptarse a los nuevos requerimientos socio-laborales del entorno (Montes y Suárez, 2016), partir de competencias (Braghirolli, Ribeiro, Weise y Pizzolato, 2016) y habilidades innovadoras como pensamiento crítico, creatividad, colaboración y comunicación (Qian y Clark, 2016), que les permitan resolver problemas y tomar decisiones en los diferentes campos de acción. Estas destrezas en ocasiones son difíciles de fomentar en las aulas de clase debido a que emplean técnicas y estrategias tradicionales que no permiten observar la eficacia de las decisiones a tomar, ni reconocer la reacción de éstas ante posibles errores o cambios del entorno (Brennan y Vos, 2013), ya que estas técnicas sólo involucran el desarrollo del área cognitiva de los estudiantes (Dávila y Velásquez, 2007).

Como alternativa de aprendizaje que permita a los estudiantes replicar el entorno de una industria en particular y proporcionar experiencia en la toma de decisiones, las clases magistrales se han apoyado en aprendizaje basado en juegos, que involucran el desarrollo de las áreas cognitiva, afectiva, emocional y conductual del estudiante, estimulando su imaginación, lo que le brinda mayor abstracción de la realidad (Dávila y Velásquez, 2007), puesto que los juegos proporcionan ambientes holísticos complejos basados en problemas, donde se recrean escenarios que permiten experimentar la responsabilidad de asumir diferentes roles bajo las mismas presiones que se enfrenta en la realidad(Brennan y Vos, 2013), a la par de ofrecerle a sus participante un ambiente colaborativo, desafiante y de retroalimentación (Qian y Clark, 2016).

Entre los campos de acción, el marketing no ha estado exento de apoyar sus clases magistrales con el aprendizaje basado en juegos (Vos, 2015), aplicándolos en temas como: contratación y capacitación de vendedores, análisis de clientes, publicidad e investigación de marketing, producción (McRaith y Goeldner, 1962), estrategias de la mezcla de marketing (Forrester, 1959; March, 1962; Shycon y Maffei, 1960), estudio del comportamiento del consumidor (Titus y Petroshius, 1993), entre otros temas; sin embargo, entre las variables de marketing encontradas en la literatura, poco énfasis se ha hecho en el diseño de juegos para promover el aprendizaje de la auditoría al proceso de marketing, a pesar de ser la que mide los resultados (en términos financieros) de las acciones de esta área (Radulescu y Cetina, 2012).

Considerando lo anterior, el objetivo de este trabajo fue diseñar el juego gerencial MKnow Audit para complementar el aprendizaje de la auditoría de marketing; en la primera parte del artículo se presenta una revisión sobre los tópicos a tratar, luego se expone la metodología propuesta por Van der Zee, Holkenborg y Robinson (2012); Prieto, López, Medina-Medina, Paderewski y Gutiérrez-Vela (2017) y Xu, Buhalis y Weber (2017), que al tener una estructura en común conforman el modelo metodológico a implementar en el diseño del juego; por último, se muestran los hallazgos de la prueba piloto, los cuales validaron que el juego complementa el proceso de aprendizaje de la auditoría de marketing.

\subsection{Aprendizaje basado en juegos}

La literatura muestra que la historia de los juegos de negocios se remonta a casi 5,000 años para el desarrollo de juegos de mesa y juegos de guerra (Faria, Hutchinson, Wellington y Gold, 2009), pero es en el siglo XIX donde toman mayor fuerza al aplicarse en la preparación de la Segunda Guerra Mundial, y al servir como insumo para el desarrollo de herramientas, como simuladores de vuelo y capacitación de personal (Lane, 1995). Con los avances tecnológicos, en 1956 la American Managment Association creó el primer juego gerencial denominado Top Management Decision Simulation, y desde entonces se han desarrollado juegos como herramientas de aprendizaje tanto en la academia como en las empresas (Mahboubian, 2010).

En términos educativos, los juegos se describen como un entorno donde tanto el contenido como el juego en sí facilitan y mejoran la adquisición de conocimiento y habilidades (Waiyakoon, Khlaisang y Koraneekij, 2015) para la resolución de problemas y desafíos, a partir del aprendizaje enfocado en el logro (Qian y Clark, 2016), donde los estudiantes aprenden de forma divertida, interesante y estimulante, (Brennan y Vos, 2013), al crear, comprender y retener conocimientos, hasta llegar al punto en que puedan 
aprender por sí mismos (Waiyakoon et al., 2015) y de forma colaborativa (Osés, Duarte y Pinto, 2016).

Ahora bien, en el campo de estudio de índole administrativa, los juegos se definen como un medio de formación con propósito educativo, que realiza una abstracción del entorno empresarial, donde los participantes del juego deben tomar decisiones relacionadas con la gestión de la empresa o de áreas específicas (Lewis y Maylor, 2007).

\subsection{Juegos gerenciales en marketing}

Los educadores que trabajan temas de marketing han implementado el uso de los juegos gerenciales en sus aulas de clase como una alternativa de aprendizaje, donde el estudiante participa en la construcción del mismo (Vos y Brennan, 2010), y a fortalecer al mismo tiempo su aprendizaje en las variables propias de la mezcla de marketing (Cronin y McCarthy, 2012), como: la distribución (Shycon y Maffei, 1960), los precios (March, 1962) y la publicidad (Forrester, 1959); o en tendencias propias del marketing, como marketing social (Russell-Bennett, Leo, Rundle-Thiele y Drennan, 2016), marketing viral (Payne, Campbell, Bal y Piercy, 2011), gestión de la marca (Craciun y Corrigan, 2009), ética de marketing, comportamiento del consumidor (Titus y Petroshius, 1993) y medio ambiente (Wiese y Sherman, 2010), entre otras variables (McRaith y Goeldner, 1962). A partir de lo anterior, se puede observar que de las variables estudiadas o encontradas en la literatura, poco énfasis se ha puesto en el proceso de marketing, especialmente en la auditoría de marketing, a pesar de ser esta herramienta la que mide el impacto de las acciones de marketing en términos financieros y de resultados (Radulescu y Cetina, 2012).

\subsection{Auditoría de marketing}

La auditoría de marketing es considerada una herramienta de evaluación que permite medir el valor, el riesgo y la eficacia de los esfuerzo de las actividades del proceso de marketing (Radulescu y Cetina, 2012), y tiene como objetivo diagnosticar, pronosticar y emitir recomendaciones (Mokwa, 1986) sobre los efectos que tienen dichos esfuerzos, así como implementar nuevas acciones que mejoren los resultados a través de diferentes alternativas de acción (Brownlie, 1993; Kotler, Gregor y Rodgers, 1989); para lo cual se han definido como instrumentos de medición diferentes indicadores de marketing relacionados con la participación de mercado, la calidad percibida, la fidelidad, la rentabilidad el precio relativo y el valor en el tiempo del cliente actual y potencial (Barwise y Farley, 2004), la satisfacción del cliente, número de quejas, la intención de compra, el conocimiento del producto y la disponibilidad del producto (Sampaio, Simões, Perin y Almeida, 2011).

Para cumplir con su objetivo, esta herramienta posee cuatro características fundamentales: comprensiva, independiente, sistemática y periódica. Se dice que es compresiva porque debe cubrir el entorno de la organización, sus objetivos, políticas, estrategias; independiente porque debe ser implementada por un agente externo al proceso de marketing para garantizar la subjetividad en el análisis (Brownlie, 1993); debe ser sistemática puesto que cumple con una secuencia ordenada de pasos que soportan el diagnóstico; y debe de ser periódica porque se realiza de forma regular (Kotler et al., 1989).

\section{Método}

El presente artículo hace parte de una práctica pedagógica aplicada a un grupo de estudiantes seleccionados a partir de un muestreo por conveniencia, donde el criterio de inclusión de los participantes fue que estuviesen cursando una asignatura que tuviera en su microcurrículo la temática de auditoría de marketing. Por lo anterior, el tipo de investigación realizada es de carácter descriptivo y cualitativo, los resultados obtenidos se interpretaron mediante un análisis descriptivo teniendo presente la conceptualización y categorización de lo observado y su coherencia con el objeto de estudio.

Para el diseño del juego se tomaron en cuenta diferentes propuestas metodológicas de algunos autores para el diseño de un juego gerencial. Por ejemplo, van der Zee et al. (2012) sugieren que para diseñar un juego es necesario definir los objetivos y parámetros, desarrollar el modelo del juego, decidir acerca de la representación del juego, construirlo y modificarlo, y por último diseñar el manual de operación. Por su 
parte Prieto et al. (2017) plantean el diseño de un videojuego educacional que contiene el diseño de los desafíos del juego con sus competencias y objetivos, el diseño del tipo de juego, el diseño inicial de la historia y los principales personajes. Una vez se define la idea general, los autores proponen refinar el juego mediante el diseño de los capítulos y las escenas, redefinir el componente educativo y emocional, ajustar el juego y finalmente decidir si el juego es individual o en grupos. Por otro lado, Xu et al. (2017) sugieren identificar las razones de uso del juego, descubrir qué aspectos motivan a jugarlo, identificar los elementos necesarios para su diseño y realizar una prueba para hacer ajustes.

En general, las metodologías revisadas proponen definir los objetivos, el modelo del juego (perfil de jugadores, roles, estructura e instrucciones), establecer los aspectos que serán evaluados, realizar una prueba piloto para hacer ajustes al diseño inicial y finalmente ser evaluado por los jugadores para determinar si se alcanzan los objetivos trazados. De acuerdo con estos elementos, un esquema metodológico para el diseño del juego MKnow Audit quedaría plasmado como lo muestra la figura 1.

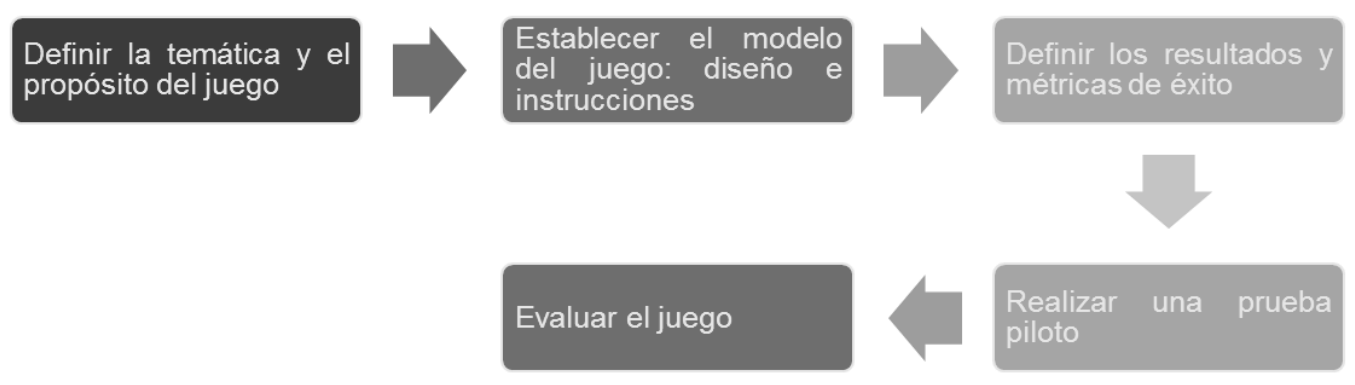

Fuente: Elaboración propia.

Figura 1. Metodología para el diseño del juego MKnow Audit

\section{Resultados}

El desarrollo del Juego gerencial Mknow Audit se dividió en cinco etapas:

1. Definición de la temática y los objetivos del juego. La temática del juego es la auditoría de marketing y el propósito es reforzar las cualidades de la auditoría de marketing: sistemática, independiente, periódica y comprensiva. El juego permite comprender la estructura de la auditoría de marketing al relacionar los objetivos de marketing con los indicadores, para que al final los participantes propongan un escenario de acción.

2. Diseño del juego e instrucciones. Los jugadores deben ser estudiantes de asignaturas que contemplen dentro de su microcurrículo la temática de auditoria de marketing, estos recibirán un documento escrito con los antecedentes de la empresa a la que aplicarán la auditoría. Una vez leído el documento cada equipo recibirá 18 piezas, con las cuales en 12 minutos construirán dos rompecabezas, uno con un escenario óptimo y otro con un escenario probable, comprobando así la característica de comprensible de la auditoría de marketing. Los jugadores sólo tendrán acceso a una clave al reverso en forma de imagen para saber si armaron el escenario óptimo o el probable. Luego, los participantes deberán discutir sobre la lógica del rompecabezas para comprender que el orden corresponde a los pasos que se llevan a cabo en el proceso de marketing en el siguiente orden: misión, visión, entorno externo, entorno interno, clientes, competencia y recursos de marketing, lo anterior comprueba la característica sistemática de la auditoría de marketing (ver figura 2). 


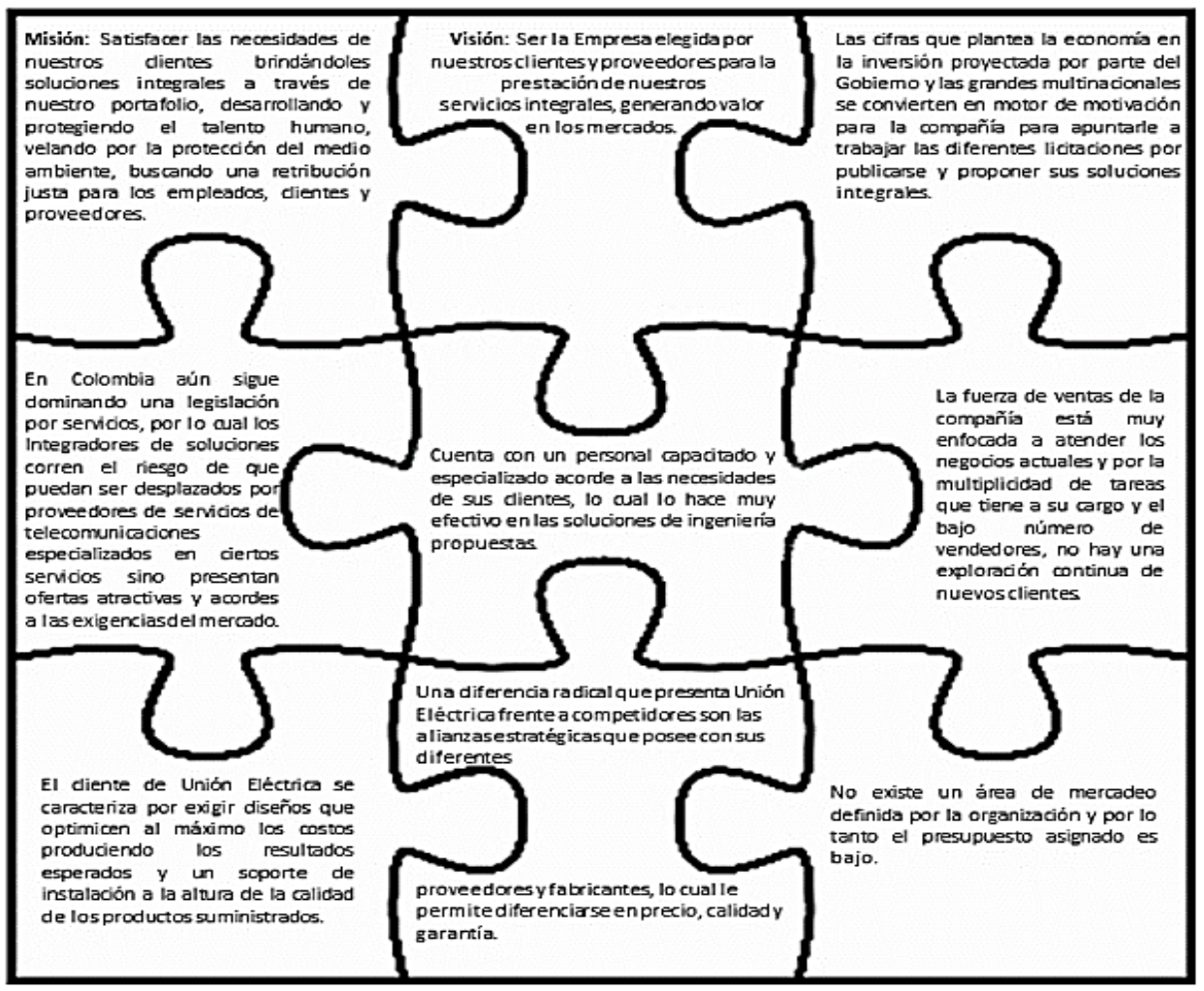

Fuente: Elaboración propia.

Figura 2. Rompecabezas con solución óptima del juego Mknow Audit

Posteriormente, a cada grupo se le entregará un juego de cartas que contiene tres objetivos de marketing identificados con la letra $\mathrm{O}$, con sus respectivos indicadores marcados con la letra I y sus resultados marcados con la letra R (ver figura 3), con el fin de que cada grupo arme en 10 minutos tres juegos de cartas con una combinación de O, I y R; sin embargo, en el grupo de cartas se presenta un juego que no guarda coherencia con el contexto de la empresa. Al igual que en el rompecabezas, se presenta un escenario óptimo y otro probable.

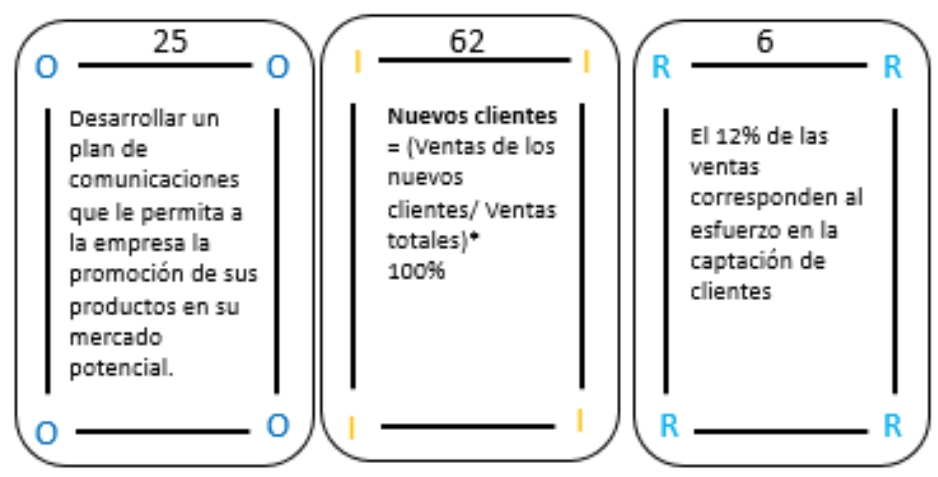

Fuente: Elaboración propia.

Figura 3. Ejemplo de un juego de cartas del juego Mknow Audit 
Una vez organizadas las cartas los jugadores deberán consignar en una hoja de respuestas sus nombres, la clave del rompecabezas y las series de números que armaron con los tres juegos de cartas, los números aparecen en la parte superior de cada carta. Los datos serán consignados en un archivo Excel para ser comparados con las respuestas dadas por los expertos. El equipo ganador es aquel que, al finalizar el juego, se acerque más al escenario óptimo propuesto por los expertos.

3. Resultados y métricas de éxito. Una vez se ingresan los resultados se asigna un puntaje automático a cada equipo de acuerdo con los siguientes criterios: el rompecabezas tiene un peso del $60 \%$ en la calificación: se asigna 5 al equipo que armó el escenario óptimo y 4 al equipo que armó el escenario probable o con fichas combinadas; los juegos de cartas tienen un peso del $40 \%$ y se calcula un promedio de calificación de cada juego de cartas: un juego de cartas armado correctamente tiene una calificación de 5 , con dos fichas correctas tiene una calificación de 3.3 y el juego de cartas que utilice alguna carta de la combinación incorrecta tiene 0.

4. Prueba piloto. La prueba piloto se aplicó a 24 estudiantes (divididos en tres grupos) del pregrado de marketing de sexto semestre en la Universidad de Medellín (Colombia).

5. Evaluación del juego. De los tres equipos conformados, sólo uno armó el rompecabezas óptimo, el cual presenta un lenguaje neutral, no plantea cursos de acción y el análisis está enmarcado con el contexto de la organización evaluada. Por su parte, los equipos que armaron el rompecabezas probable mantuvieron el orden lógico de las dimensiones de la auditoría. Las principales dificultades se presentaron en los juegos de cartas donde cada equipo presentó sólo un juego de cartas acertado, los participantes manifestaron confusión por no leer detenidamente el alcance de los objetivos para determinar cuáles son los indicadores y resultados que guardan mayor coherencia. Cuando fueron justificadas las combinaciones de cartas acertadas por parte del coordinador del juego, los participantes comprendieron que los errores radican en analizar el cumplimiento de los objetivos de marketing desde un punto de vista operativo y no estratégico.

Como parte de la metodología, los estudiantes evaluaron el juego en una hoja de respuestas adicional, respondiendo preguntas referentes al diseño, la complejidad, el aprendizaje, las estrategias y los aspectos de mejora del juego. Estos resultados se agruparon en dos categorías: una relacionada con las características del juego y otra con el aprendizaje a través del juego. En la primera categoría los participantes sugieren que la imagen posterior del rompecabezas debe presentar alta dificultad para que los jugadores se concentren en armar la cara que contiene el orden lógico de los resultados de una auditoría de marketing.

Mientras los resultados de cada equipo de jugadores son reportados en Excel es necesario que el juego continúe, por lo cual se propone que reporten en una hoja de respuestas adicional un escenario de acción para la empresa auditada de acuerdo con la información que analizaron. El escenario de acción propuesto por los equipos de jugadores se compara con el escenario propuesto por un grupo de expertos, lo que permite el cierre del análisis y desempatar puntajes para determinar al equipo ganador.

En la segunda categoría de resultados, a partir de una pregunta abierta, los participantes manifiestan los aspectos aprendidos en el juego. En los resultados se identifican dos líneas de aprendizaje, una relacionada con condiciones implícitas en una auditoría y otra con aspectos propios de la auditoría de marketing. En la primera línea resalta que hay muchos caminos para obtener soluciones (17\%), la importancia de llevar un orden lógico (13\%) y de asociar y conectar información (13\%) (ver tabla I), habilidades que los estudiantes deben fortalecer en su proceso de aprendizaje para el desarrollo de las competencias que propone el programa de formación. Por otro lado, la segunda línea destaca que la auditoría de marketing soporta una evaluación extensa a las organizaciones (8\%), que el juego permite reconocer los elementos de la auditoría (8\%) y aspectos más específicos como el análisis del entorno y desde la perspectiva de los objetivos de marketing (8\%) 
Tabla I. Aspectos aprendidos en el juego

\begin{tabular}{l|l|r}
\hline Calificación & Línea & Frec. \% \\
\hline Hay muchos caminos para solucionar las cosas & Auditoría & 17 \\
Llevar un orden lógico & Auditoría & 13 \\
Asociar y conectar información & Auditoría & 13 \\
La evaluación extensa que se le puede hacer a una empresa & Auditoría de marketing & 8 \\
Elementos para una auditoría & Auditoría de marketing & 8 \\
La forma como se presentan los resultados y la evaluación de una auditoría & Auditoría de marketing & 8 \\
A leer las instrucciones & Auditoría & 8 \\
Mejorar la habilidad de análisis & Auditoría & 8 \\
Secuencia, interpretación y análisis & Auditoría & 8 \\
Analizar el entorno & Auditoría de marketing & 4 \\
Analizar desde la perspectiva de los objetivos & Auditoría de marketing & 4 \\
\hline
\end{tabular}

Por su parte, la tabla II evidencia las estrategias que los estudiantes consideran claves para ganar el juego y que guardan amplia relación con los objetivos del juego, como son: la importancia de leer bien las instrucciones (25\%), trabajar en equipo (21\%), aplicar la lógica (12.5\%), conocer el orden de una auditoría (12.5\%) y realizar un análisis profundo (12.5\%).

Tabla II. Estrategia para ganar el juego

\begin{tabular}{l|c|c}
\hline Calificación & $\#$ & Frec. \% \\
\hline Leer bien las instrucciones & 6 & 25.0 \\
Trabajo en equipo & 5 & 20.8 \\
La lógica & 3 & 12.5 \\
El orden para realizar una auditoría & 3 & 12.5 \\
Análisis profundo & 3 & 12.5 \\
Planificación & 1 & 4.2 \\
Leer bien la información de la auditoría & 1 & 4.2 \\
Relacionar bien la información & 1 & 4.2 \\
Relacionar los conceptos & 1 & 4.2 \\
Armar las figuras de atrás y luego buscar la lógica & 1 & 4.2 \\
Tener claridad de la información de la empresa & 1 & 4.2 \\
Tener conocimientos previos & 1 & 4.2 \\
\hline \multicolumn{2}{|c}{}
\end{tabular}

\section{Conclusiones}

La posibilidad de que los jugadores pasen por un proceso de comunicación intra e intergrupal exige desarrollar destrezas como llevar un orden lógico, asociar y conectar información, y mejorar la habilidad de análisis -elementos vitales para la formación administrativa y gerencial de los estudiantes. Además, la importancia de leer bien las instrucciones, trabajar en equipo, aplicar la lógica, tener un breve conocimiento del tema y realizar un análisis profundo son tareas que los jugadores consideran clave para ganar el juego y guardan amplia relación con los objetivos del mismo.

En la revisión de literatura se evidenció que el uso de los juegos gerenciales en el área de auditoría de marketing es incipiente, lo que permite presentar un juego gerencial enfocado en esta área de formación, que facilita al estudiante medir con indicadores el desempeño del proceso de marketing, lo que deriva en oportunidades para fortalecer las estrategias de aprendizaje en el aula. 
El juego gerencial MKnow Audit complementa el proceso de aprendizaje de la auditoría de marketing porque evalúa de forma dinámica sus cuatro características: comprensiva, independiente, sistemática y periódica.

\section{Referencias}

Barwise, P. y Farley, J. U. (2004). Marketing metrics: status of six metrics in five countries. European Management Journal, 22(3), 257-262. doi:10.1016/j.emj.2004.04.012

Braghirolli, L., Ribeiro, J. L., Weise, A. D. y Pizzolato, M. (2016). Benefits of educational games as an introductory activity in industrial engineering education. Computers in Human Behavior, 58, 315-324. doi:10.1016/j.chb.2015.12.063

Brennan, R. y Vos, L. (2013). Effects of participation in a simulation game on marketing students' numeracy and financial skills. Journal of Marketing Education, 35(3), 259-270.

doi:10.1177/0273475313482928

Brownlie, D. T. (1993). The marketing audit: a metrology and explanation. Marketing Intelligence and Planning, 11(1), 4-12. doi:10.1108/02634509310024128

Craciun, G. y Corrigan, H. (2009). An Integrative experiential learning project in the undergraduate branding course: creating a marketing department brochure. Journal of Marketing Education, 32(2), 116127. doi:10.1177/0273475309344808

Cronin, J. y McCarthy, M. (2012). Marketing "gamer foods"?: qualitative insights into responsible strategy development. Journal of Food Products Marketing, 18(3), 163-185. doi:10.1080/10454446.2012.666448

Dávila, G. y Velásquez, Á. (2007). Evaluación de la aplicación de juegos colaborativos: "Devorón" y "Temporal". Revista Electrónica de Investigación Educativa, 9(2), 1-20. Recuperado de https://redie.uabc.mx/redie/article/view/164/283

Faria, A. J., Hutchinson, D., Wellington, W. J. y Gold, S. (2009). Developments in business gaming?: a review of the past 40 years. Simulation and Gaming, 40(4), 464-487. doi:1046878108327585

Forrester, J. W. (1959). Advertising: a problem in industrial dynamics. Harvard Business Review, 37(2), 100110.

Kotler, P., Gregor, W. y Rodgers, W. (1989). The marketing audit comes of age. MITSloan Management Review, 18(2), 25-43. Recuperado de https://sloanreview.mit.edu/article/the-marketing-audit-comes-ofage/

Lane, D. C. (1995). On a resurgence of management simulations and games. The Journal of the Operational Research Society, 46(5), 604-625. doi:10.2307/2584534

Lewis, M. A. y Maylor, H. R. (2007). Game playing and operations management education. International Journal of Production Economics, 105(1), 134-149. doi:10.1016/j.ijpe.2006.02.009

Mahboubian, M. (2010). Educational aspects of business simulation softwares. Procedia-Social and Behavioral Sciences, 2(2), 5403-5407. doi:10.1016/j.sbspro.2010.03.881

March, J. (1962). The business firm a political coalition. The Journal of Politics, 24(4), 662-678. doi:10.1017/S0022381600016169

McRaith, J. F. y Goeldner, C. R. (1962). A survey of marketing games. Journal of Marketing, 26(3), 69-72. 
doi:10.2307/1248306

Mokwa, M. P. (1986). The strategis marketing audit: an adoption/utilization perspect. Journal of Business Strategy, 6(4), 88-95. doi:10.1108/eb039136

Montes, D. A. y Suárez, C. I. (2016). La formación docente universitaria: claves formativas de universidades españolas. Revista Electrónica de Investigación Educativa, 18(3), 53-61. Recuperado de http://redie.uabc.mx/redie/article/view/996

Osés, R. M., Duarte, E. y Pinto, M. L. (2016). Juegos cooperativos: efectos en el comportamiento asertivo en niños de 60. grado de escuelas públicas. Revista Electrónica de Investigación Educativa, 18(3), 176-186. Recuperado de http://redie.uabc.mx/redie/article/view/886

Payne, N. J., Campbell, C., Bal, A. S. y Piercy, N. (2011). Placing a hand in the fire: assessing the impact of a YouTube experiential learning project on viral marketing knowledge acquisition. Journal of Marketing Education, 33(2), 204-216. doi:10.1177/0273475311410853

Prieto, R., López, J. R., Medina-Medina, N., Paderewski, P. y Gutiérrez-Vela, F. L. (2017). Design methodology foreducational games based on graphical notations: designing Urano. Entertainment Computing, 18, 1-14. doi:10.1016/j.entcom.2016.08.005

Qian, M. y Clark, K. R. (2016). Game-based learning and 21st century skills: a review of recent research. Computers in Human Behavior, 63, 50-58. doi:1016/j.chb.2016.05.023

Radulescu, V. y Cetina, I. (2012). customer analysis, defining component of marketing audit. ProcediaSocial and Behavioral Sciences, 62, 308-312. doi:10.1016/j.sbspro.2012.09.050

Russell-Bennett, R., Leo, C., Rundle-Thiele, S. y Drennan, J. (2016). A hierarchy-of-effects approach to designing a social marketing game. Journal of Nonprofit and Public Sector Marketing, 28(2), 105-128.

doi:10.1080/10495142.2014.988081

Sampaio, C. H., Simões, C., Perin, M. G. y Almeida, A. (2011). Marketing metrics: insights from brazilian managers. Industrial Marketing Management, 40(1), 8-16. doi:10.1016/j.indmarman.2010.09.005

Shycon, H. N. y Maffei, R. B. (1960). Simulation tool for better distribution. Harvard Business Review, 38(6), 65-75.

Titus, P. A. y Petroshius, S. M. (1993). Bringing Consumer behavior to the workbench: an experiential approach. Journal of Marketing Education, 15(1), 20-30. doi:10.1177/027347539301500104

Van der Zee, D. J., Holkenborg, B. y Robinson, S. (2012). Conceptual modeling for simulation-based serious gaming. Decision Support Systems, 54(1), 33-45. doi:10.1016/j.dss.2012.03.006

Vos, L. (2015). Simulation games in business and marketing education: How educators assess student learning from simulations. The International Journal of Management Education, 13(1), 57-74.

doi:10.1016/j.ijme.2015.01.001

Vos, L. y Brennan, R. (2010). Marketing simulation games: student and lecturer perspectives. Marketing Intelligence and Planning, 28(7), 882-897. doi:10.1108/02634501011086472

Waiyakoon, S., Khlaisang, J. y Koraneekij, P. (2015). Development of an instructional learning object design model for tablets using game-based learning with scaffolding to enhance mathematical concepts for mathematic learning disability students. Procedia - Social and Behavioral Sciences, 174, 1489-1496.

doi:10.1016/j.sbspro.2015.01.779 
Wiese, N. M. y Sherman, D. J. (2010). Integrating marketing and environmental studies through an interdisciplinary, experiential, service-learning approach. Journal of Marketing Education, 33(1), 41-56. doi:10.1177/0273475310389154

Xu, F., Buhalis, D. y Weber, J. (2017). Serious games and the gamification of tourism. Tourism Management, 60(C), 244-256. doi:10.1016/j.tourman.2016.11.020 